\title{
Predictive ability of direct genomic values for lifetime net merit of Holstein sires using selected subsets of single nucleotide polymorphism markers
}

\author{
K. A. Weigel, ${ }^{* 1}$ G. de los Campos,† O. González-Recio,‡ H. Naya,§ X. L. Wu, ${ }^{*}$ N. Long,† G. J. M. Rosa,† \\ and D. Gianola*†\# \\ *Department of Dairy Science, and \\ †Department of Animal Sciences, University of Wisconsin, Madison 53706 \\ ‡Departamento de Mejora Genética, Instituto Nacional de Investigaciones Agrarias, Madrid, Spain \\ $\S B$ Bioinformatics Unit, Institut Pasteur de Montevideo, Uruguay \\ \#Department of Biostatistics and Medical Informatics, University of Wisconsin, Madison 53706
}

\begin{abstract}
The objective of the present study was to assess the predictive ability of subsets of single nucleotide polymorphism (SNP) markers for development of low-cost, low-density genotyping assays in dairy cattle. Dense SNP genotypes of 4,703 Holstein bulls were provided by the USDA Agricultural Research Service. A subset of 3,305 bulls born from 1952 to 1998 was used to fit various models (training set), and a subset of 1,398 bulls born from 1999 to 2002 was used to evaluate their predictive ability (testing set). After editing, data included genotypes for 32,518 SNP and August 2003 and April 2008 predicted transmitting abilities (PTA) for lifetime net merit (LNM $\$)$, the latter resulting from progeny testing. The Bayesian least absolute shrinkage and selection operator method was used to regress August 2003 PTA on marker covariates in the training set to arrive at estimates of marker effects and direct genomic $\mathrm{PTA}$. The coefficient of determination $\left(\mathrm{R}^{2}\right)$ from regressing the April 2008 progeny test PTA of bulls in the testing set on their August 2003 direct genomic PTA was 0.375 . Subsets of $300,500,750,1,000,1,250,1,500$, and 2,000 SNP were created by choosing equally spaced and highly ranked SNP, with the latter based on the absolute value of their estimated effects obtained from the training set. The SNP effects were re-estimated from the training set for each subset of SNP, and the 2008 progeny test PTA of bulls in the testing set were regressed on corresponding direct genomic PTA. The $\mathrm{R}^{2}$ values for subsets of 300,500,750, 1,000, 1,250, 1,500, and 2,000 SNP with largest effects (evenly spaced SNP) were $0.184(0.064), 0.236(0.111), 0.269$ (0.190), 0.289 (0.179), 0.307 (0.228), $0.313(0.268)$, and 0.322 (0.291), respectively. These results indicate that a low-density assay comprising selected SNP could be a cost-effective
\end{abstract}

Received January 30, 2009

Accepted June 30, 2009

${ }^{1}$ Corresponding author: kweigel@wisc.edu alternative for selection decisions and that significant gains in predictive ability may be achieved by increasing the number of SNP allocated to such an assay from 300 or fewer to 1,000 or more.

Key words: single nucleotide polymorphism, lifetime net merit, predicted transmitting ability, genotyping

\section{INTRODUCTION}

Recent advances in technologies for genotyping individual animals, most notably the development of highthroughput assays for dense genotyping of SNP (Van Tassell et al., 2008), have led to a flurry of research and development activity in the area of whole-genome selection of livestock. The foundation was laid by Meuwissen et al. (2001), who showed via simulation that correlations between simulated breeding values and genomeenhanced predicted breeding values were nearly twice what could be achieved using parent averages (PA) in the absence of SNP data. Numerous methods have been proposed for estimating SNP effects and predicting genome-enhanced PTA of individual animals, but a basic linear model for computing direct genomic PTA from dense SNP genotypes can be described thus:

$$
y_{i}=\mu+\sum_{j=1}^{p} x_{i j} \beta_{j}+e_{i}
$$

where $\mu$ is a parameter common to all observations and the $\beta_{j}$ 's represent the regressions of $y_{i}$ on SNP covariates $\left(x_{i j}, j=1,2, \ldots p\right)$. In nearly every case, these SNP need not be the causal mutation and do not represent QTL with direct effects on the phenotype of interest; they may be simply markers in noncoding regions of the genome that are in linkage disequilibrium (LD) with neighboring QTL. It is generally assumed that these QTL act additively, such that the $\beta_{j}$ 's refer to per-allele effects. Nonadditive effects and the effects of unmarked QTL are lumped together in the residual term, $e_{i}$, or captured as additive effects under certain 
circumstances, thereby accounting for most of the additive and nonadditive genetic variance (Hill et al., 2008). Because the number of markers may exceed the number of individuals, fitting such a model using standard regression methods is not trivial, even if only additive effects are considered. To overcome this problem, several alternatives have been proposed, including dimension-reduction techniques, stepwise procedures, nonparametric regression, and ridge regression (e.g., Whittaker et al., 2000; Hastie et al., 2001; Gianola et al., 2006; Muir, 2007; Gianola and van Kaam, 2008). Another alternative is a hierarchical Bayesian modeling approach in which the $\beta_{j}$ 's are assumed to be random effects distributed according to some prespecified distribution; for example, one may assume that the $\beta_{j}$ 's are normally distributed with mean 0 and variance $\sigma_{j}^{2}$ or extend the hierarchy by assigning a prior distribution to each of the variances $\sigma_{j}^{2}$ (e.g., Meuwissen et al., 2001; Gianola et al., 2003; Xu, 2003; ter Braak et al., 2005).

VanRaden et al. (2009) recently demonstrated the possibility of enhancing genetic progress in the North American bovine AI industry through the use of genomic information. Genotypes for 39,835 SNP were combined with progeny phenotypes, which were recorded in 2003, from 2,609 Holstein bulls that were born from 1954 to 1997. Estimated SNP effects for economically important dairy traits were combined with traditional PA to produce genome-enhanced PTA. Subsequently, predictive ability was assessed by comparing genome-enhanced PTA for 510 Holstein bulls that were born from 2001 to 2003 with their progeny phenotypes, which were recorded in 2008. The realized gains in empirical reliability (REL) for each trait from the inclusion of genomic information ranged from 5 to $34 \%$, with larger gains for traits with known major genes (e.g., fat percentage). The average increase in REL across all traits was $18 \%$ in the study of VanRaden et al. (2009), although gains in REL are presently much larger because of a 2 -fold increase in the number of US Holstein sires with dense SNP genotypes and progeny phenotypes (P. M. VanRaden, USDA-ARS, Beltsville, MD; personal communication).

At the current price, tools such as the Illumina BovineSNP50 BeadChip (Illumina Inc., San Diego, CA) may be limited to applications involving males and elite females. A low-cost assay with between 300 and 1,000 SNP might deliver a substantial portion of the gain for a small fraction of the price. Applications could include preliminary screening of young bulls, selection of replacement heifers, discovery of parentage (when pedigree data are missing or errant), selection of mates for specific cows, or development of management protocols that reflect the genetic predispositions of individual animals. However, the optimal number of SNP for a lowdensity assay is unknown, as is the appropriate strategy for selecting the SNP to be included in such an assay. It is likely that the optimal solution will depend on the genetic architecture underlying the traits of interest, the recent population and family structure and amount of LD in the species of interest, the proportion of ancestors with high-density SNP genotypes, and the genetic relationships between individuals used to estimate SNP effects (i.e., the training set) and future candidates for genomic selection (i.e., the testing set).

The objective of this study was to evaluate the potential of low-cost, low-density SNP genotyping assays for use in genetic selection programs by evaluating the predictive ability of direct genomic PTA for lifetime net merit (LNM\$) that were derived from subsets of selected SNP or equally spaced SNP of various size.

\section{MATERIALS AND METHODS}

\section{Data}

High-density SNP genotypes, which were derived from the Illumina BovineSNP50 BeadChip, and sire PTA for LNM\$, which were the result of progeny testing, were obtained from the Bovine Functional Genomics Laboratory and Animal Improvement Programs Laboratory, respectively, at the USDA-ARS Beltsville Agricultural Research Center (Beltsville, MD). Models were fitted to a training set comprising dense SNP genotypes and August 2003 progeny test PTA for LNM $\$$ of 3,305 Holstein bulls that were born from 1952 to 1998. Mean theoretical REL of August 2003 progeny test PTA for LNM $\$$ of 3,305 bulls in the training set was $84.2 \%$, and $3,209,2,771$, and 517 of these bulls exceeded 70, 80, and $90 \%$ theoretical REL, respectively. The predictive ability of the models fitted was evaluated using a testing set comprising dense SNP genotypes and April 2008 progeny test PTA for LNM $\$$ of 1,398 Holstein bulls that were born from 1999 to 2002. In the testing set, mean theoretical REL of August 2003 PA for LNM\$ and April 2008 progeny test PTA for LNM $\$$ of bulls were 35.0 and $81.8 \%$, respectively. Of the 1,398 bulls in the testing set, 1,195 (85.5\%) were sired by bulls in the training set, and 991 (70.9\%) had maternal grandsires that were present in the training set.

Genotypes at each locus were coded as 0 (homozygous for allele A), 1 (heterozygous), 2 (homozygous for allele B), or 5 (missing). The 38,416 SNP used by VanRaden et al. (2009), which represented a reduction from the original 51,386 SNP that could be read reliably due to removal of SNP with minor allele frequency $<0.05$ and SNP that were in complete LD with adjacent SNP, 
were available for the present study. Slightly less than $1 \%$ of SNP genotypes were missing and, in this case, information from flanking markers was used to compute the conditional probability of each possible genotype, and the most probable value was subsequently chosen for imputation. Although this approach greatly reduced the number of missing genotypes, we also removed the sires $(\mathrm{n}=230)$ and SNP $(\mathrm{n}=5,898)$ that had the greatest number of missing genotypes after imputation to generate the final data set of $32,518 \mathrm{SNP}$ and 4,703 sires that was used in the analyses described herein.

\section{Estimation of Marker Effects}

The PTA of sires in the training set were regressed on marker covariates using the Bayesian LASSO (least absolute shrinkage and selection operator; BL) of Park and Casella (2008). This model is a Bayesian formulation of Tibshirani's (1996) LASSO method. The probability model of the BL is defined as follows:

$$
\begin{gathered}
\text { Likelihood : } p\left(\mathbf{y} \mid \boldsymbol{\beta}, \sigma^{2}\right)=\prod_{i=1}^{n} N\left(\mathbf{y}_{i} \mid \mathbf{x}_{i}^{\prime} \boldsymbol{\beta}, \sigma^{2}\right) ; \\
\text { Prior : } p\left(\boldsymbol{\beta}, \sigma^{2}, \boldsymbol{\tau}^{2}, \lambda\right)=p\left(\boldsymbol{\beta} \mid \sigma^{2}, \boldsymbol{\tau}^{2}\right) p\left(\sigma^{2}\right) p\left(\boldsymbol{\tau}^{2} \mid \lambda\right) p(\lambda) \\
=\left\{\prod_{j=1}^{p} N\left(\beta_{j} \mid 0, \sigma^{2} \boldsymbol{\tau}_{j}^{2}\right)\right\} \\
\chi^{-2}\left(\sigma^{2} \mid d f, S\right) \operatorname{Exp}\left(\boldsymbol{\tau}_{j}^{2} \mid \lambda\right) G\left(\lambda \mid \alpha_{1}, \alpha_{2}\right),
\end{gathered}
$$

where $N\left(\mathbf{y}_{i} \mid \mathbf{x}_{i}^{\prime} \boldsymbol{\beta}, \sigma^{2}\right)$ and $N\left(\beta_{j} \mid 0, \sigma^{2} \boldsymbol{\tau}_{j}^{2}\right)$ are normal density functions centered at $\mathbf{x}_{j}^{\prime} \boldsymbol{\beta}$ and 0 , with variances $\sigma^{2}$ and $\sigma^{2} \boldsymbol{\tau}_{j}^{2}$, respectively; $\chi^{-2}\left(\sigma^{2} \mid d f, S\right)$ is a scaled inverted-chi-square distribution with degrees of freedom $d f$ and inverted scale parameter $S ; \operatorname{Exp}\left(\tau_{j}^{2} \mid \lambda\right)$ is an exponential distribution assigned to a positive scale parameter $\tau_{j}^{2} ; \lambda$ is a regularization parameter for the shape of the prior distribution assigned to the $\boldsymbol{\tau}_{j}^{2}$; and $G\left(\lambda \mid \alpha_{1}, \alpha_{2}\right)$ is a gamma distribution with shape and rate parameters $\alpha_{1}$ and $\alpha_{2}$, respectively. All fully conditional distributions have closed form, and a Gibbs sampler can be used to draw samples from the posterior distribution. Details of the BL method, as applied to genetic selection of livestock, can be found in de los Campos et al. (2009).

The aforementioned BL method was used to estimate SNP effects by fitting the model to the training set using all the available markers $(32,518)$ as predictor variables and August 2003 progeny test PTA for
LNM $\$$, standardized to have a sample variance equal to 1 , as the response variable. These estimated SNP effects were used for 2 purposes:

1) To evaluate the predictive ability of a highdensity assay. Estimates from the model fitted to the training set were used to compute direct genomic PTA for the bulls in the testing set, and these estimates were compared with their August 2008 progeny test PTA for LNM $\$$. The criteria for comparison included the coefficient of determination $\left(\mathrm{R}^{2}\right)$ from linear regression, and the proportion of selected sires that were in common.

2) To select different subsets of SNP to be included in a low-density assay. Subsets of SNP with "largest" effects were created by sorting all 32,518 SNP by the absolute values of the posterior means of their estimated effects (from largest to smallest) and then choosing the top 300, 500, $750,1,000,1,250,1,500$, or 2,000 SNP. Subsets of equally spaced SNP were created by sorting all 32,518 SNP by chromosome and by position within chromosome and then choosing the first SNP and every $1 / 300,1 / 500,1 / 750,1 / 1,000$, $1 / 1,250,1 / 1,500$, or $1 / 2,000$ of the $32,518 \mathrm{SNP}$ thereafter.

Subsequently, SNP effects were recomputed by repeating the BL analysis, using SNP genotypes and August 2003 PTA for LNM $\$$ of bulls in the training set, for each of the 14 subsets; that is, the 7 subsets based on the largest effect criterion plus the 7 subsets based on equal spacing of markers. Next, the direct genomic PTA of bulls in the testing set, which were computed using estimates of marker effects from the training set and genotypes of bulls in the testing set, were compared with their August 2008 progeny test PTA for LNM\$ using the criteria described above.

All of the BL analyses were implemented via the Gibbs sampler using version 2.8.0 of R (R Foundation for Statistical Computing, 2008). A detailed description of the methodology is provided in de los Campos et al. (2009). The following specifications were assumed for prior distributions: $\mathrm{df}=10, S=\frac{1}{2}, \alpha_{1}=\frac{1}{30}$, and $\alpha_{2}=3$. These values give priors that are flat over a wide range of relevant values of the corresponding parameters. A chain of 110,000 samples was run, and the first 10,000 samples were discarded as burn-in. Posterior summaries were computed using a thinning rate of 50. Convergence was checked by visual inspection of trace plots. 
Table 1. Percentage of selected SNP (300, 750, 1,250, or 2,000 SNP) for lifetime net merit on each of the Bos taurus autosomes (BTA) and X chromosome based on equal spacing (EQ) or Bayesian Lasso solutions (BL) from a training set comprising genotypes and daughter phenotypes for 3,305 Holstein bulls born from 1952 to 1998

\begin{tabular}{lccccccccc}
\hline Location & EQ 300 & BL 300 & EQ 750 & BL 750 & EQ 1,250 & BL 1,250 & EQ 2,000 & BL 2,000 & All 32,518 \\
\hline BTA1 & 6.0 & 3.7 & 5.9 & 3.5 & 5.9 & 3.8 & 5.9 & 4.2 & 5.9 \\
BTA2 & 4.7 & 5.0 & 4.9 & 5.6 & 4.9 & 5.4 & 5.0 & 5.0 & 4.9 \\
BTA3 & 5.0 & 2.7 & 4.8 & 5.9 & 4.8 & 4.9 & 4.8 & 4.4 & 4.8 \\
BTA4 & 4.7 & 1.7 & 4.7 & 2.3 & 4.6 & 2.8 & 4.6 & 3.8 & 4.6 \\
BTA5 & 4.0 & 3.0 & 4.0 & 3.3 & 4.0 & 4.2 & 4.1 & 4.2 & 4.0 \\
BTA6 & 4.3 & 1.7 & 4.5 & 2.4 & 4.6 & 3.1 & 4.6 & 2.8 & 4.6 \\
BTA7 & 4.3 & 3.7 & 4.1 & 5.1 & 4.2 & 4.7 & 4.2 & 4.7 & 4.1 \\
BTA8 & 4.3 & 1.7 & 4.5 & 1.5 & 4.5 & 1.8 & 4.5 & 2.0 & 4.5 \\
BTA9 & 3.7 & 3.3 & 3.7 & 3.5 & 3.8 & 4.4 & 3.8 & 4.6 & 3.8 \\
BTA10 & 4.3 & 1.7 & 4.1 & 2.4 & 4.2 & 2.7 & 4.2 & 3.1 & 4.1 \\
BTA11 & 4.3 & 5.0 & 4.3 & 4.8 & 4.2 & 4.7 & 4.3 & 4.3 & 4.2 \\
BTA12 & 3.0 & 3.0 & 3.1 & 3.1 & 3.1 & 3.2 & 3.1 & 3.3 & 3.1 \\
BTA13 & 3.3 & 5.7 & 3.5 & 4.5 & 3.4 & 3.5 & 3.4 & 3.6 & 3.4 \\
BTA14 & 3.3 & 4.3 & 3.2 & 4.0 & 3.3 & 4.1 & 3.3 & 3.6 & 3.3 \\
BTA15 & 3.3 & 4.3 & 3.3 & 3.9 & 3.3 & 3.3 & 3.3 & 3.1 & 3.3 \\
BTA16 & 2.7 & 1.0 & 2.8 & 1.5 & 2.8 & 1.5 & 2.8 & 2.0 & 2.8 \\
BTA17 & 3.0 & 1.0 & 2.9 & 2.4 & 3.0 & 2.6 & 3.0 & 3.0 & 3.0 \\
BTA18 & 2.7 & 8.3 & 2.7 & 5.2 & 2.7 & 5.0 & 2.7 & 4.6 & 2.7 \\
BTA19 & 2.7 & 3.7 & 2.7 & 1.9 & 2.7 & 2.6 & 2.8 & 2.6 & 2.7 \\
BTA20 & 3.0 & 3.7 & 2.9 & 2.9 & 2.8 & 3.3 & 2.8 & 3.0 & 2.8 \\
BTA21 & 2.3 & 3.7 & 2.5 & 3.1 & 2.6 & 3.4 & 2.6 & 3.2 & 2.5 \\
BTA22 & 2.0 & 2.0 & 2.5 & 2.4 & 2.5 & 2.3 & 2.5 & 2.4 & 2.5 \\
BTA23 & 2.7 & 2.7 & 2.1 & 2.4 & 2.2 & 2.2 & 2.2 & 2.5 & 2.2 \\
BTA24 & 2.0 & 5.0 & 2.4 & 3.7 & 2.4 & 3.1 & 2.4 & 2.9 & 2.4 \\
BTA25 & 2.3 & 3.7 & 2.0 & 3.2 & 2.0 & 2.8 & 2.1 & 3.0 & 2.0 \\
BTA26 & 2.0 & 2.0 & 2.0 & 1.9 & 2.0 & 2.2 & 2.0 & 2.2 & 2.0 \\
BTA27 & 2.0 & 1.3 & 1.9 & 1.3 & 1.9 & 1.5 & 1.9 & 1.8 & 1.9 \\
BTA28 & 2.0 & 2.0 & 1.9 & 2.4 & 1.8 & 2.3 & 1.9 & 2.4 & 1.9 \\
BTA29 & 2.0 & 2.7 & 2.0 & 2.8 & 2.1 & 2.3 & 2.1 & 2.4 & 2.0 \\
X & 2.3 & 2.0 & 2.4 & 3.3 & 2.4 & 2.8 & 2.4 & 2.8 & 2.4 \\
Unknown & 1.7 & 5.0 & 1.6 & 4.0 & 1.5 & 3.4 & 1.6 & 3.0 & 1.5 \\
\hline & & & & & & & & & \\
\hline
\end{tabular}

\section{RESULTS AND DISCUSSION}

To conserve space and limit redundancy, results shown (Table 1 and Figures 2 and 3) are restricted to the analyses of subsets of $300,750,1,250$, and 2,000 SNP. The chromosomal locations of SNP selected from the training set based on equal spacing or based on estimates from the BL analysis are shown in Table 1. Representation of chromosomes in the equally spaced subsets, in which SNP were ordered by chromosome and by location within chromosome (if known), was proportional to the distribution of SNP across chromosomes in the full set of 32,518 SNP.

When SNP were selected based on the magnitude of $\mathrm{BL}$ estimates in the training set, Bos taurus autosome (BTA) 18 was represented most heavily in the subsets of 300 SNP and was among the 5 most heavily represented chromosomes in every subset. Likewise, BTA2 was represented most heavily in subsets of $\geq 1,250 \mathrm{SNP}$ and was among the 5 most heavily represented chromosomes in every subset. Bos taurus autosome 3 was represented most heavily in the subset of 750 SNP and, along with BTA7 and BTA11, was among the 5 most commonly represented chromosomes in the majority of subsets. As shown in Table 1, the percentage of SNP chosen from each chromosome when selection was based on the magnitude of BL estimates was substantially different from that of equally spaced SNP.

Table 2 shows parameter estimates from the BL analysis of the training set, using data from all 32,518 SNP. The estimate of the regularization parameter $(\lambda)$ for the shape of the prior distribution assigned to the $\tau_{j}^{2}$ parameters was 112.2. As $\lambda$ approaches zero, estimates of the BL tends toward ordinary least squares, whereas large values of $\lambda$ increase the penalty on the $L_{1}$ norm of $\boldsymbol{\beta}$, thereby shrinking estimates of effects more strongly toward zero. Following the BL analysis of all $32,518 \mathrm{SNP}$, subsets of $300,500,750,1,000,1,250$, 1,500 , or 2,000 SNP were chosen based on the magnitude of their estimated effects. Subsequently, the BL analysis was repeated for each subset. This step is important, because the magnitude of the BL estimate for a given SNP can be heavily influenced by the presence or absence of neighboring SNP that are in LD with the SNP of interest. Estimates of $\lambda$ from analyses of selected subsets of SNP ranged from 20.0 to 46.4, and estimates tended to decrease as the number of SNP in 
Table 2. Parameter estimates for the regularization parameter for the shape of the prior distribution $(\lambda)$ and the estimated residual variance $\left(\sigma^{2}\right)$ for lifetime net merit derived from a training set comprising SNP genotypes and daughter phenotypes for 3,305 Holstein bulls born from

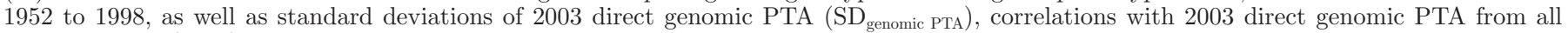
available SNP $\left(\mathrm{r}_{32518}\right)$, and correlations between 2008 progeny test PTA and 2003 direct genomic PTA for subsets of SNP with the largest estimated effects or subsets of equally spaced SNP of various size in a testing set comprising 1,398 Holstein bulls born from 1999 to 2002 ( $\mathrm{r}_{\mathrm{PT}}$ All $)$, as well as subsets of 1,195 bulls whose sires were in the training set $\left(\mathrm{r}_{\mathrm{PT}}\right.$ Sire $)$ and 203 bulls whose sires were not in the training set ( $\mathrm{r}_{\mathrm{PT}}$ NoSire)

\begin{tabular}{|c|c|c|c|c|c|c|c|c|}
\hline No. of SNP & Method of SNP selection & $\lambda$ & $\sigma^{2}$ & $\mathrm{SD}_{\text {genomic PTA }}$ & $\mathrm{r}_{32518}$ & $\mathrm{r}_{\mathrm{PT} \_\mathrm{All}}$ & $\mathrm{r}_{\mathrm{PT} \_ \text {Sire }}$ & $\mathrm{r}_{\mathrm{PT} \_ \text {NoSire }}$ \\
\hline 300 & Largest effects & 20.0 & 0.460 & 0.629 & 0.633 & 0.428 & 0.447 & 0.312 \\
\hline 300 & Equally spaced & 26.1 & 0.744 & 0.382 & 0.326 & 0.253 & 0.262 & 0.202 \\
\hline 500 & Largest effects & 24.2 & 0.350 & 0.692 & 0.716 & 0.485 & 0.503 & 0.369 \\
\hline 500 & Equally spaced & 29.8 & 0.629 & 0.463 & 0.476 & 0.333 & 0.348 & 0.245 \\
\hline 750 & Largest effects & 28.0 & 0.263 & 0.734 & 0.772 & 0.519 & 0.530 & 0.441 \\
\hline 750 & Equally spaced & 35.0 & 0.579 & 0.493 & 0.559 & 0.435 & 0.450 & 0.348 \\
\hline 1,000 & Largest effects & 31.9 & 0.210 & 0.759 & 0.822 & 0.537 & 0.549 & 0.460 \\
\hline 1,000 & Equally spaced & 37.6 & 0.540 & 0.514 & 0.611 & 0.422 & 0.438 & 0.321 \\
\hline 1,250 & Largest effects & 35.3 & 0.174 & 0.776 & 0.852 & 0.554 & 0.567 & 0.461 \\
\hline 1,250 & Equally spaced & 39.7 & 0.491 & 0.551 & 0.652 & 0.477 & 0.489 & 0.395 \\
\hline 1,500 & Largest effects & 38.4 & 0.155 & 0.786 & 0.870 & 0.559 & 0.576 & 0.445 \\
\hline 1,500 & Equally spaced & 42.4 & 0.477 & 0.561 & 0.702 & 0.518 & 0.534 & 0.412 \\
\hline 2,000 & Largest effects & 42.9 & 0.110 & 0.812 & 0.900 & 0.567 & 0.582 & 0.469 \\
\hline 2,000 & Equally spaced & 46.4 & 0.412 & 0.567 & 0.748 & 0.539 & 0.559 & 0.408 \\
\hline 32,518 & All available & 112.2 & 0.087 & 0.759 & - & 0.612 & 0.627 & 0.511 \\
\hline
\end{tabular}

the BL analysis decreased. This indicates that the removal of thousands of uninformative SNP leads to a distribution of estimates with thicker tails and less mass at zero. Furthermore, for a given number of SNP, estimates of $\lambda$ tended to be greater for the subsets of equally spaced SNP than for the subsets of SNP with largest estimated effects. This is not surprising, as analyses of the former type will tend to have a greater proportion of SNP with negligible effects.

As shown in Table 2, the estimated residual variance $\left(\sigma^{2}\right)$ was 0.087 in the BL analysis of all $32,518 \mathrm{SNP}$ in the training set, whereas this estimate ranged from 0.110 to 0.744 in the BL analyses of selected subsets of SNP. Estimates of $\sigma^{2}$ tended to decrease as the number of SNP in the analysis increased. This may imply that a greater proportion of the phenotypic variance is captured by models with a larger number of SNP, but over-fitting may occur in such models because of the large number of explanatory variables. Nonetheless, and more importantly, estimates of $\sigma^{2}$ for a subset of given size were from 1.6- to 3.7-fold greater for the analyses based on evenly spaced subsets of SNP, indicating poorer model fit.

As shown in Table 2, the standard deviation (SD) of direct genomic PTA for bulls in the testing set from the analysis of all $32,518 \mathrm{SNP}$ was 0.759 . The corresponding SD from BL analyses of subsets of SNP that were selected based on magnitude of their effects ranged from 0.629 to 0.812 and tended to increase as the number of SNP in the model increased. The aforementioned SD were 40 to $60 \%$ larger than the SD of direct genomic PTA from corresponding analyses of evenly spaced SNP, indicating a lack of power to differentiate bulls based on genetic merit in the analyses of equally spaced SNP.

Correlations between direct genomic PTA from selected subsets of SNP and direct genomic PTA from the BL analysis of all 32,518 SNP for bulls in the testing set ranged from 0.326 to 0.900 , as shown in Table 2. As expected, correlations increased as the number of SNP in the subset increased. Furthermore, correlations from subsets of SNP that were selected based on magnitude of their effects were from 20 to $90 \%$ larger than those from subsets of evenly spaced SNP. In addition, the rate of increase in correlations, with respect to size of the subset of SNP, was much more rapid for evenly spaced SNP. This indicates that the accuracy of direct genomic PTA from subsets of $\leq 500$ evenly spaced SNP will be much poorer than the accuracy of direct genomic PTAs from subsets of $\geq 1,500$ evenly spaced SNP.

The relationship between direct genomic PTA for bulls in the testing set, computed as the sum of estimated SNP effects (times the appropriate code for each SNP genotype) from the training set, and their April 2008 progeny test PTA is shown in Table 2 and Figures 1 and 2 . For the BL analysis of all $32,518 \mathrm{SNP}$, the coefficient of determination $\left(\mathrm{R}^{2}\right)$ from regression of April 2008 progeny test PTA on the corresponding direct genomic PTA was 0.375 . By comparison, the corresponding $\mathrm{R}^{2}$ for the subsets of SNP with largest estimated effects ranged from 0.184 to 0.322 , and the corresponding $\mathrm{R}^{2}$ for subsets of evenly spaced SNP ranged from 0.064 to 0.291 . Predictive ability was always greater for subsets of SNP with largest estimated effects, relative to subsets of equally spaced SNP of equivalent size. Predictive ability was particularly poor for subsets of $\leq 500$ evenly 


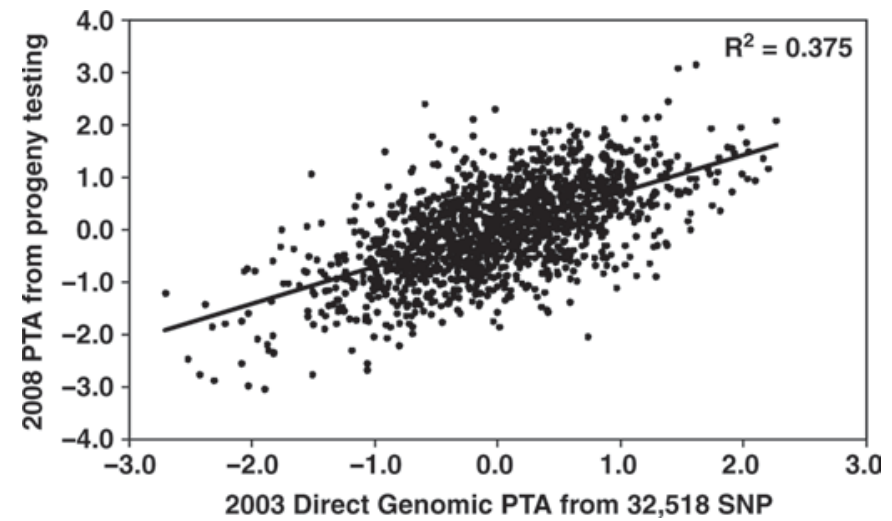

Figure 1. Relationship between 2008 progeny test PTA for lifetime net merit (in standard deviation units) and 2003 direct genomic PTA in a testing set comprising 1,398 Holstein bulls born from 1999 to 2002, using all SNP markers with estimated SNP effects derived from a training set comprising 3,305 Holstein bulls born from 1952 to 1998.

spaced SNP, but as the number of SNP increased, the difference in $\mathrm{R}^{2}$ between subsets of $\mathrm{SNP}$ with largest effects and subsets of equally spaced SNP decreased. Based on the results shown in Table 2 and Figure 2, one can speculate that a low-cost assay based on $300 \mathrm{SNP}$ may provide roughly half of the gain in REL that could be achieved with a high-density assay, whereas a lowcost assay with 750 to 1,000 SNP may provide roughly two-thirds of the gain in REL that could be achieved with a high-density assay. It is important to note that the estimates of predictive ability reported herein are specific to the population structure considered in the present study, specifically with respect to level of population-wide LD and the availability of high-density genotypes for sires, but not for dams. In particular, results shown in Table 2 indicate that the correlation between April 2008 progeny test PTA and August 2003 direct genomic PTA are substantially greater for bulls in the testing set $(\mathrm{n}=1,195)$ whose sires were represented in the training set than for bulls $(\mathrm{n}=203)$ whose sires were not represented, regardless of the number of SNP or method of SNP selection. As noted in the previous section, the April 2008 progeny test PTA of sires in the testing set had less than perfect reliability. Therefore, the $R^{2}$ values and correlations reported herein should be considered as conservative estimates of the ability of direct genomic PTA to predict the actual breeding values of these sires.

Last, the ability of direct genomic PTA to provide useful information for selection decisions, as measured by the proportion of sires selected from the testing set based on direct genomic PTA that were in common with those selected based on 2008 progeny test PTA, is shown in Table 3 and Figure 3. Selection based on direct genomic PTA from the analysis of all 32,518 SNP led to 306 of the top 500 bulls that were in common (out of 1,398 total bulls in the testing set) with respect to selection based on subsequent progeny test PTA. Selection based on direct genomic PTA from the subsets of SNP with largest effects generally led to more consistent decisions with respect to ensuing progeny test results. As the number of SNP in the analysis increased, the proportion of selected bulls that were in common with those chosen based on subsequent progeny test results increased only modestly. However, as selection intensity increased, the ability of direct genomic PTA from low- or high-density assays to identify bulls that were in common with those selected based on subsequent progeny test results declined markedly. Therefore, it is important to recognize that, at present, direct genomic PTA can be useful for identifying groups of young

Table 3. Number of bulls selected from a testing set comprising 1,398 Holstein bulls born from 1999 to 2002 based on 2008 progeny test PTA for lifetime net merit that were in common with selection based on 2003 direct genomic PTA using all 32,518 SNP, subsets of SNP with the largest estimated effects, or subsets of equally spaced SNP of various size, with estimated SNP effects derived from a training set comprising 3,305 Holstein bulls born from 1952 to 1998

\begin{tabular}{|c|c|c|c|c|c|c|c|}
\hline No. of SNP & Method of SNP selection & Top 500 bulls & Top 250 bulls & Top 100 bulls & Top 50 bulls & Top 25 bulls & Top 10 bulls \\
\hline 300 & Largest effects & 272 & 89 & 20 & 7 & 1 & 0 \\
\hline 300 & Equally spaced & 230 & 64 & 13 & 4 & 1 & 0 \\
\hline 500 & Largest effects & 289 & 94 & 24 & 6 & 2 & 1 \\
\hline 500 & Equally spaced & 247 & 81 & 22 & 4 & 1 & 0 \\
\hline 750 & Largest effects & 292 & 99 & 22 & 8 & 3 & 1 \\
\hline 750 & Equally spaced & 268 & 91 & 18 & 5 & 2 & 1 \\
\hline 1,000 & Largest effects & 299 & 102 & 22 & 5 & 2 & 1 \\
\hline 1,000 & Equally spaced & 271 & 92 & 14 & 4 & 0 & 0 \\
\hline 1,250 & Largest effects & 307 & 101 & 23 & 8 & 2 & 1 \\
\hline 1,250 & Equally spaced & 280 & 105 & 28 & 6 & 1 & 0 \\
\hline 1,500 & Largest effects & 304 & 109 & 22 & 9 & 2 & 1 \\
\hline 1,500 & Equally spaced & 285 & 99 & 25 & 9 & 2 & 0 \\
\hline 2,000 & Largest effects & 308 & 109 & 25 & 11 & 3 & 2 \\
\hline 2,000 & Equally spaced & 292 & 103 & 21 & 6 & 2 & 1 \\
\hline 32,518 & All available & 306 & 114 & 27 & 9 & 4 & 1 \\
\hline
\end{tabular}




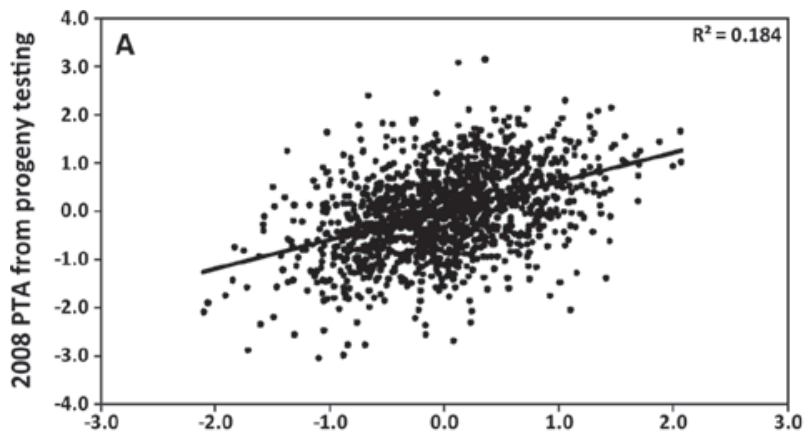

2003 Direct Genomic PTA from 300 SNP with Largest Effects

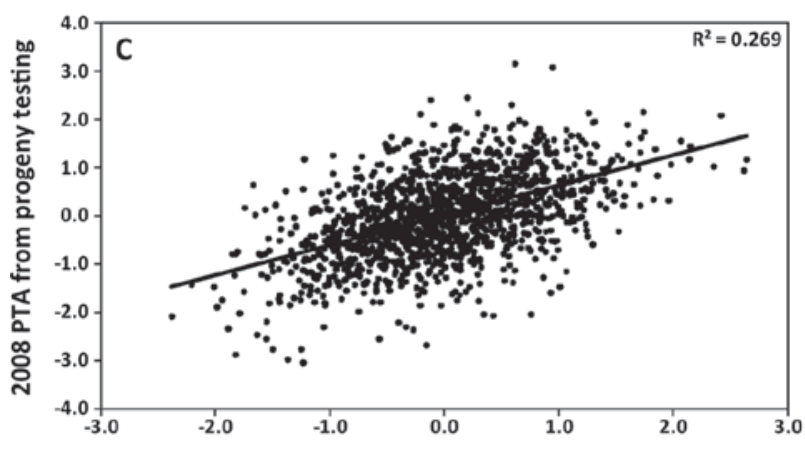

2003 Direct Genomic PTA from 750 SNP with Largest Effects

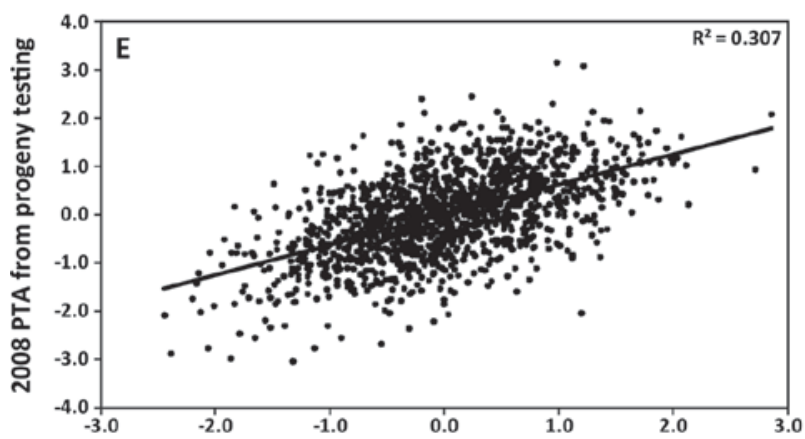

2003 Direct Genomic PTA from 1250 SNP with Largest Effects

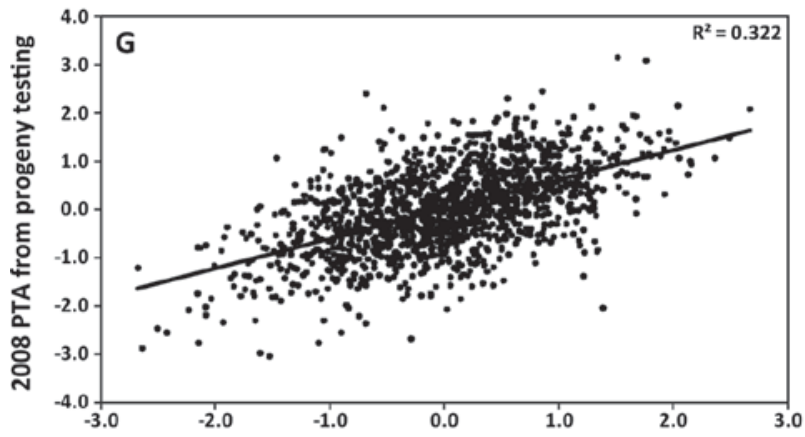

2003 Direct Genomic PTA from 2000 SNP with Largest Effects
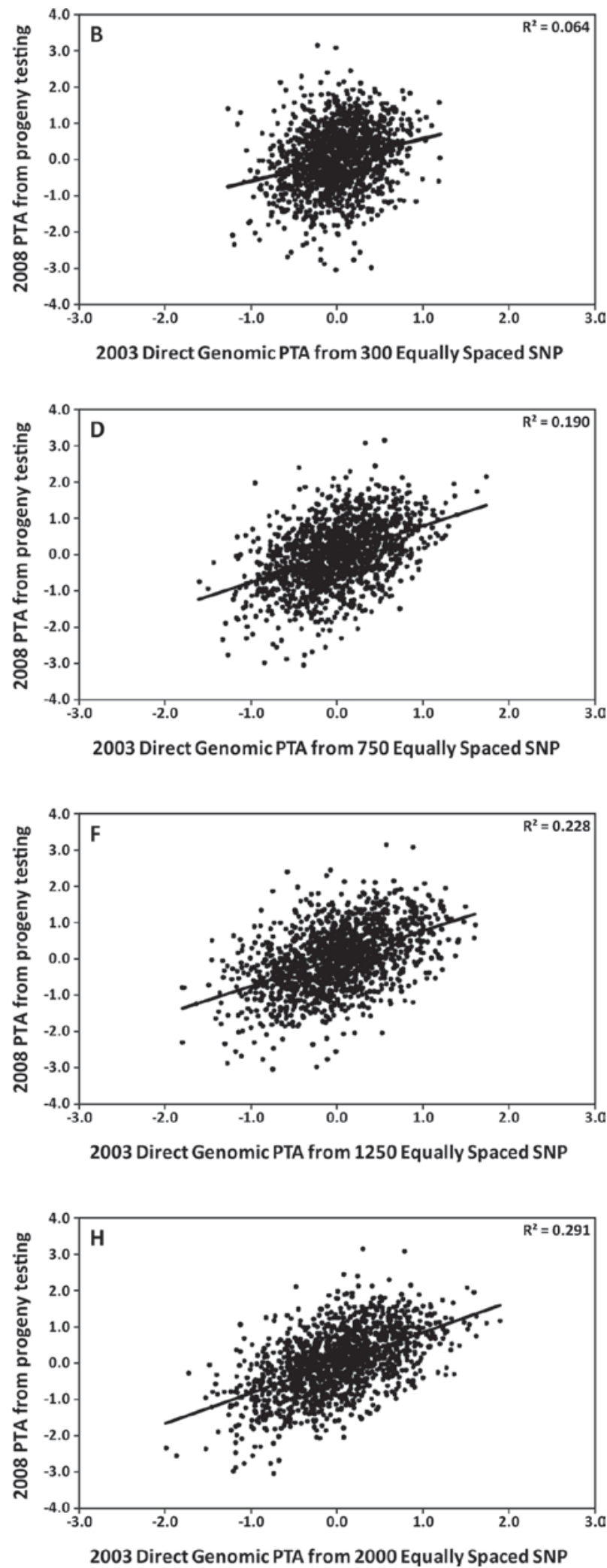

Figure 2. Relationship between 2008 progeny test PTA for lifetime net merit (in standard deviation units) and 2003 direct genomic PTA in a testing set comprising 1,398 Holstein bulls born from 1999 to 2002, using subsets of SNP with the largest estimated effects: A) 300 SNP, C) $750 \mathrm{SNP}, \mathrm{E})$ 1,250 SNP, G) 2,000 SNP, or subsets of equally spaced SNP: B) $300 \mathrm{SNP}, \mathrm{D}) 750 \mathrm{SNP}, \mathrm{F})$ 1,250 SNP, H) 2,000 SNP, with estimated SNP effects derived from a training set comprising 3,305 Holstein bulls born from 1952 to 1998. 


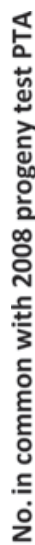

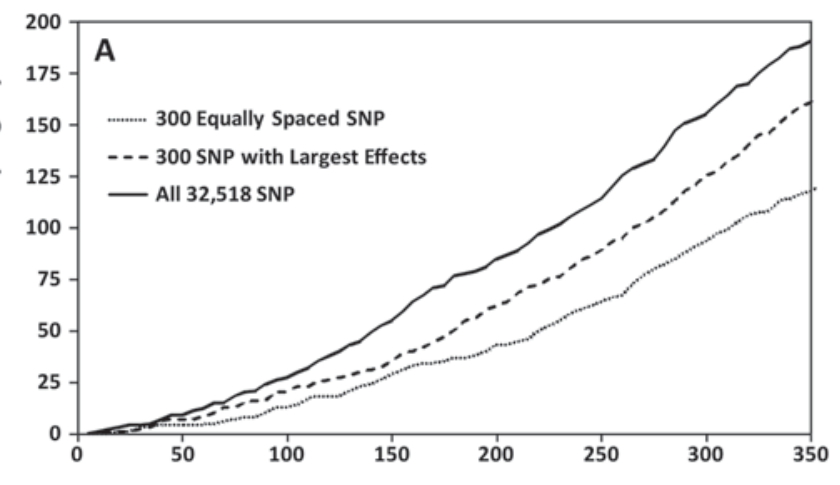

No. Bulls Selected based on 2003 Direct Genomic PTA

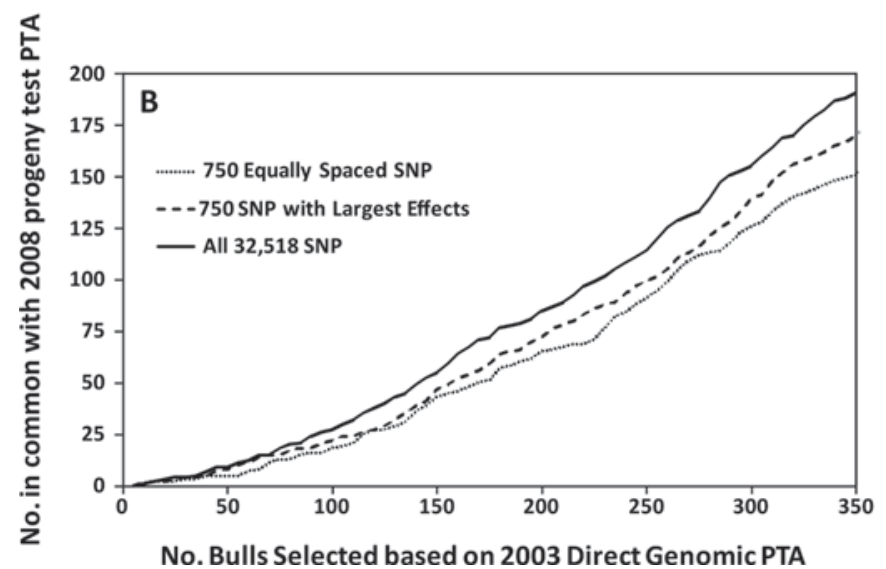

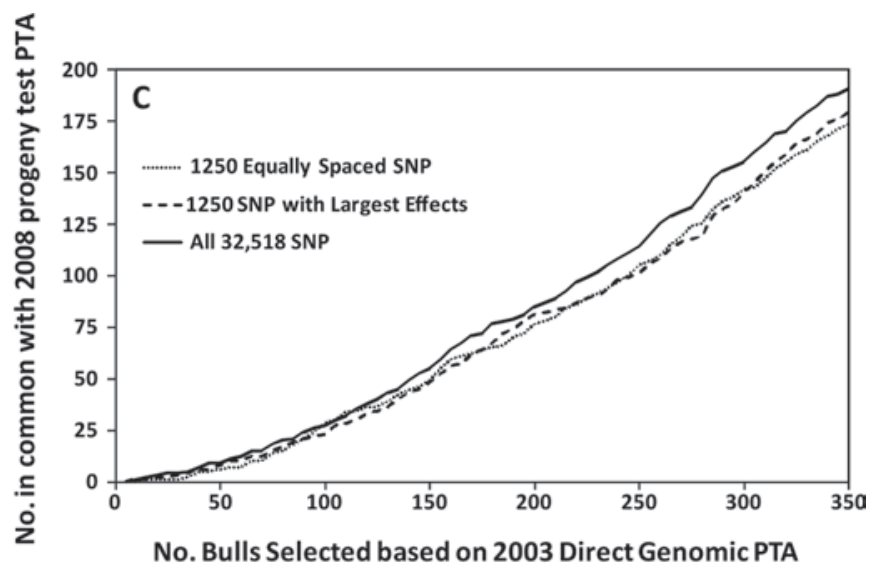

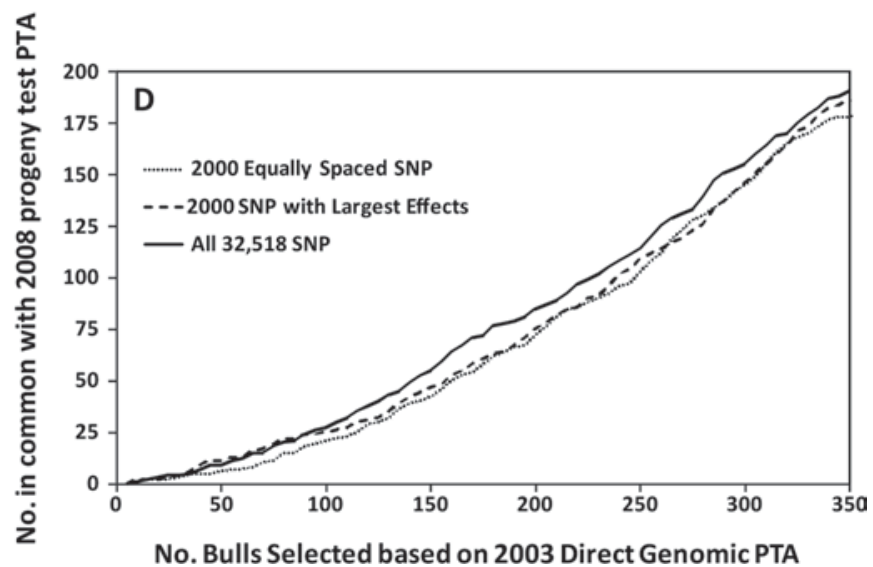

Figure 3. Number of bulls selected from a testing set comprising 1,398 Holstein bulls born from 1999 to 2002 based on 2008 progeny test PTA for lifetime net merit that were in common with selection based on 2003 direct genomic PTA using all 32,518 SNP, subsets of SNP with the largest estimated effects, or subsets of equally spaced SNP: A) $300 \mathrm{SNP}$, B) $750 \mathrm{SNP}, \mathrm{C})$ 1,250 SNP, or D) 2,000 SNP, with estimated SNP effects derived from a training set comprising 3,305 Holstein bulls born from 1952 to 1998.

animals that are likely to rank in the top one-half or one-quarter of their cohorts, but the predictive ability of direct genomic PTA from low- or high-density assays is not yet sufficient for precise ranking of individual animals within these selected groups. Again, it is important to note that the April 2008 progeny test PTA had imperfect reliability, and selection based on breeding values with reliability near unity may have yielded more favorable results. Moreover, sire PTA may not be the optimal response variable in genomic selection, because it is a regressed and smoothed product of a model with strict assumptions. Exploration of adjusted progeny mean as a response variable may be of interest in future studies.

It is important to note that the present study considered only direct genomic PTA, which were computed by summing estimated SNP effects, rather than genome-enhanced PTA, which include both pedigree and genomic information. In future studies, it would be desirable to include pedigree data via the additive relationship matrix, as described by de los Campos et al. (2009), because it is likely that this additional information would enhance predictive ability.

As noted by Habier et al. (2008), low-density assays with evenly spaced SNP may be very effective in situations in which both parents are routinely genotyped using a high-density assay, because reconstruction of parental haplotypes may be possible. Such a tool could be useful in nucleus breeding herds, because dense genotyping of valuable, elite breeding stock can be easily justified. It is likely that dense genotyping of potential bull dams on commercial dairy farms will soon become commonplace as well, so a low-density assay with evenly spaced markers may be useful for selection of young dairy bulls and elite, high-value cows, heifers, and calves.

\section{CONCLUSIONS}

Although it is likely that whole-genome selection of elite males and females based on dense SNP genotypes will greatly enhance genetic progress in cattle, swine, 
and poultry, the current cost of such assays precludes routine genotyping of a large number of individuals on commercial farms. Results of the present study indicate that a low-density assay comprising a subset of SNP with the largest estimated effects on LNM\$ may provide useful information for selection decisions. An assay comprising 300 SNP might capture nearly half of the gain in REL that could be achieved by dense genotyping, particularly for animals whose sires are represented in the training population that is used to estimate SNP effects, whereas a low-cost assay with 750 to 1,000 SNP may provide roughly two-thirds of the gain in REL that can be achieved with a high-density assay. Results of the present study reflect the predictive ability of direct genomic PTA derived from the specific training and testing sets of Holstein sires described herein, and the performance of direct genomic PTA or genome-enhanced PTA computed from selected subsets of SNP is likely to differ in other populations. In addition, selection of SNP in the present study was based on magnitude of the estimated marker effects, but in the future one might consider selection of SNP based on their expected contributions to the genetic variance in previous generations, which is a function of allele frequencies in the training population, or their expected contributions to the genetic variance in the next generation, which is a function of allele frequencies in the training population (which may or may not be known at the time of SNP selection). Potential applications include selection of replacement heifers (particularly on farms that utilize sex-enhanced semen), preliminary screening of young bulls or potential bull dams (followed by dense genotyping of selected animals), and discovery of parentage.

In the future, it is likely that breeding companies will develop multiple low-density genotyping assays. As noted earlier, an assay comprising equally spaced SNP may be optimal in situations in which both parents have dense genotypic data, whereas an assay comprising selected SNP with large effects may be optimal in situations where only sires have dense genotypic data. To the extent that breeding goals differ between companies, different subsets of SNP should be selected. Furthermore, different subsets of SNP may be appropriate for selection under different management or environmental conditions. Finally, the subsets of SNP chosen for applications involving genomic selection, which will reflect additive genetic variation, may not be optimal for applications such as selection of optimal mates or development of customized management protocols, because these should consider both additive and nonadditive variation in the performance of commercial cows. Perhaps the most challenging aspect of widespread SNP genotyping using low-density assays will be the development of efficient mechanisms for collecting, shipping, and processing tens of thousands of DNA samples from hundreds of commercial farms.

\section{ACKNOWLEDGMENTS}

The authors gratefully acknowledge scientists at the USDA-ARS Beltsville Agricultural Research Center for proving genotypic and phenotypic data for the present study. In addition, the technical expertise of C. P. Van Tassell, P. M. VanRaden, and J. R. O'Connell of the USDA-ARS (Beltsville, MD) is greatly appreciated. K. Weigel acknowledges financial support from the National Association of Animal Breeders (Columbia, MO), and O. González-Recio acknowledges financial support from the UW-Madison Babcock Institute for International Dairy Research and Development. This project was supported by National Research Initiative Grant no. 2009-35205-05099 from the USDA Cooperative State Research, Education, and Extension Service.

\section{REFERENCES}

de los Campos, G., H. Naya, D. Gianola, J. Crossa, A. Legarra, E. Manfredi, K. Weigel, and J. M. Cotes. 2009. Predicting quantitative traits with regression models for dense molecular markers. Genetics 182:375-385.

Gianola, D., R. L. Fernando, and A. Stella. 2006. Genomic assisted prediction of genetic values with semi-parametric procedures. Genetics 173:1761-1776.

Gianola, D., M. Perez-Enciso, and M. Toro. 2003. On marker-assisted prediction of genetic value: Beyond the ridge. Genetics 163:347365.

Gianola, D., and J. B. C. H. M. van Kaam. 2008. Reproducing kernel Hilbert spaces regression methods for genomic assisted prediction of quantitative traits. Genetics 178:2289-2303.

Habier, D., J. C. M. Dekkers, and R. L. Fernando. 2008. Genomic selection using low-density SNPs. J. Anim. Sci. 86(E. Suppl. 2):506. (Abstr.)

Hastie, T., R. Tibshirani, and J. H. Friedman. 2001. The Elements of Statistical Learning: Data Mining, Inference, and Predictions. Springer-Verlag Inc., New York, NY.

Hill, W. G., M. E. Goddard, and P. M. Visscher. 2008. Data and theory point to mainly additive genetic variance for complex traits. PLoS Genet. 4:e1000008.

Meuwissen, T. H. E., B. J. Hayes, and M. E. Goddard. 2001. Prediction of total genetic value using genome-wide dense marker maps. Genetics 157:1819-1829.

Muir, W. M. 2007. Genomic selection, accuracy and comparisons with traditional BLUP under alternative marker density and generations of training. J. Anim. Breed. Genet. 6:342-355.

Park, T., and G. Casella. 2008. The Bayesian Lasso. J. Am. Stat. Assoc. 103:681-686.

R Foundation for Statistical Computing. 2008. Version 2.8.0. http:// www.r-project.org/

ter Braak, C. J. F., M. P. Boer, and M. C. A. M. Bink. 2005. Extending Xu's Bayesian model for estimating polygenic effects using markers of the entire genome. Genetics 170:1435-1438.

Tibshirani, R. 1996. Regression shrinkage and selection via the Lasso. J. R. Stat. Soc., B 58:267-288.

Van Tassell, C. P., T. P. L. Smith, L. K. Matukumalli, J. F. Taylor, R. D. Schnabel, C. T. Lawley, C. D. Haudenschild, S. S. Moore, W. 
C. Warren, and T. S. Sonstegard. 2008. SNP discovery and allele frequency estimation by deep sequencing of reduced representation libraries. Nat. Methods 5:247-252.

VanRaden, P. M., C. P. Van Tassell, G. R. Wiggans, T. S. Sonstegard, R. D. Schnabel, and F. Schenkel. 2009. Reliability of genomic predictions for North American dairy bulls. J. Dairy Sci. 92:1624.
Whittaker, J. C., R. Thompson, and M. C. Denham. 2000. Markerassisted selection using ridge regression. Genet. Res. Camb. 75:249-252.

$\mathrm{Xu}$, S. 2003. Estimating polygenic effects using markers of the entire genome. Genetics 163:789-801. 